\title{
Management of rectal gastrointestinal stromal tumor
}

\author{
Hitoshi Kameyama ${ }^{1}$, Tatsuo Kanda ${ }^{2}$, Yosuke Tajima ${ }^{1}$, Yoshifumi Shimada ${ }^{1}$, Hiroshi Ichikawa ${ }^{1}$, Takaaki \\ Hanyu $^{1}$, Takashi Ishikawa ${ }^{1}$, Toshifumi Wakai ${ }^{1}$ \\ ${ }^{1}$ Division of Digestive and General Surgery, Niigata University Graduate School of Medical and Dental Sciences, Niigata, Japan; ${ }^{2}$ Department of \\ Surgery, Sanjo General Hospital, Niigata, Japan \\ Contributions: (I) Conception and design: All authors; (II) Administrative support: None; (III) Provision of study materials or patients: None; \\ (IV) Collection and assembly of data: None; (V) Data analysis and interpretation: None; (VI) Manuscript writing: All authors; (VII) Final approval of \\ manuscript: All authors. \\ Correspondence to: Hitoshi Kameyama. Division of Digestive and General Surgery, Niigata University Graduate School of Medical and Dental \\ Sciences, 1-757 Asahimachi-dori, Chuo-ku, Niigata City, 951-8510, Japan. Email: kame@med.niigata-u.ac.jp.
}

\begin{abstract}
Gastrointestinal stromal tumor (GIST) is the most common mesenchymal neoplasm of the gastrointestinal tract. However, rectal GIST is rare, the incident rate of it is approximately $5 \%$ of all GISTs. Rectal GIST symptoms generally include bleeding and/or pain and occasionally, urinary symptoms. Immunohistochemical evaluation finds that most rectal GIST tumors are CD117 (KIT) positive, and are sometimes CD34, platelet-derived growth factor receptor alpha (PDGFRA), smooth muscle actin, S-100, or vimentin positive. The National Institutes of Health (NIH) classifies rectal GIST as very-low risk, low risk, intermediate risk, or high risk, and the frequencies have been estimated as $0-23.8 \%$ for very-low risk, $0-45 \%$ for low risk, $0-34 \%$ for intermediate risk, and $21-100 \%$ for high risk tumors. The first-line treatment for localized GIST is curative resection, but is difficult in rectal GIST because of anatomical characteristics such as the deep, narrow pelvis and proximity to the sphincter muscle or other organs. Several studies noted the efficacy of the minimally invasive surgery, such as trans-anal, trans-sacral, trans-vaginal resection, or laparoscopic resection. The appropriate surgical procedure should be selected depending on the case. Imatinib mesylate (IM) is indicated as first-line treatment of metastatic or unresectable GIST, and clinical outcomes are correlated with KIT mutation genotype. However, the KIT mutation genotypes in rectal GIST are not well known. In this review, as in other GISTs, a large proportion (59-100\%) of rectal GISTs carry exon 11 mutations. Although curative resection is indicated for localized rectal GIST, a high rate of local recurrence is a problem. Multimodal therapy including perioperative IM may improve postoperative outcomes, contributing to anus-preserving surgery. Moreover, KIT mutation analysis before IM treatment is important. This review summarizes current treatment strategies for rectal GIST.
\end{abstract}

Keywords: Gastrointestinal stromal tumor (GIST); rectal GIST; KIT mutation; imatinib mesylate (IM)

Received: 24 November 2017; Accepted: 16 January 2018; Published: 01 February 2018.

doi: $10.21037 /$ tgh.2018.01.08

View this article at: http://dx.doi.org/10.21037/tgh.2018.01.08

\section{Introduction}

Gastrointestinal stromal tumors (GISTs) were first described by Mazur et al. in 1983 (1). GISTs are the most common mesenchymal neoplasms of the gastrointestinal tract and can have spindle-cell or epithelioid histology; $80 \%$ express the KIT protein and $10 \%$ express platelet-derived growth factor receptor alpha (PDGFRA) (2,3). Gain-of-function mutations in the KIT proto-oncogene or PDGFRA are important in the genesis and classification of these tumors (3-6). These mutations are involved in GIST development, and result in the constitutive activation of KIT signaling (4). GISTs account for $0.1-3 \%$ of all malignant gastrointestinal neoplasms (7-9), and rectal GIST is rare, with an incidence of approximately $0.1 \%$ of all rectal neoplasms (10), and 
Table 1 Clinical symptoms of rectal GIST

\begin{tabular}{|c|c|c|c|c|c|c|c|c|c|}
\hline Authors & Year & $\begin{array}{c}\text { No. of } \\
\text { patients }\end{array}$ & Pain & Bleeding & $\begin{array}{l}\text { Change of } \\
\text { bowel habit }\end{array}$ & Constipation & Tumor & $\begin{array}{l}\text { Urinary } \\
\text { symptom }\end{array}$ & Others \\
\hline Hamada (22) & 2008 & 33 & $3.0 \%$ & $30.3 \%$ & - & $15.2 \%$ & $12.1 \%$ & $3.3 \%$ & $36.1 \%$ \\
\hline Agaimy (25) & 2013 & 15 & - & $46.7 \%$ & - & - & $53.3 \%$ & $13.3 \%$ & - \\
\hline Pai (23) & 2015 & 13 & $38.5 \%$ & $23.0 \%$ & - & - & - & - & $38.5 \%$ \\
\hline Wilkinson (26) & 2015 & 19 & $21.0 \%$ & $36.8 \%$ & $31.6 \%$ & - & - & - & $31.6 \%$ \\
\hline
\end{tabular}

GIST, gastrointestinal stromal tumor.

comprises approximately 5\% of all GISTs (11).

Curative resection is the first-line treatment for localized GISTs in all organs, but is difficult in rectal GIST because of anatomical features including the deep, narrow pelvis and proximity to the sphincter muscle or other organs. Several studies have reported the efficacy of multimodality therapy for rectal GIST, including perioperative imatinib mesylate (IM) treatment. Laparoscopic surgery or anus-preserving surgery for rectal GIST preserves patient quality of life (QOL). This review discusses the current treatment of rectal GIST.

\section{Epidemiology}

GIST may occur anywhere in the gastrointestinal tract, but its frequency is mostly in the stomach (60-70\%) followed by small intestine $(25-30 \%)$, rectum $(5 \%)$, and colon (1\%) (12). Furthermore, GIST may also occur as primary tumors outside of the gastrointestinal tract proper as intra-abdominal locations in the mesenteries, omentum, retroperitoneum, or pelvis (12-14). Colorectal GIST was reported to account for $6.3 \%$ of cases in western Sweden (15), and other studies reported that rectal GIST accounted for $3.5-5 \%$ of all cases (15-17). The annual incidence of GIST is approximately $1.1-1.45$ per 100,000 population $(15,17)$ and the overall incidence has been estimated as $10-20$ per 100,000 population including incidental, minimal tumors (18). Rectal GIST accounts for $4 \%$ of all GISTs, or 800-1,000 new cases in the European Union each year $(15,17,19)$. Hawkins et al. found that 333 anorectal GIST patients were registered in the National Cancer Database, that their mean age was 62.3 years, and that the median tumor size was $4.0 \mathrm{~cm}$ (20).

Rectal GISTs are also rare in eastern countries, accounting for approximately $0.1 \%$ of all rectal neoplasms in South Korea (10). Yasui et al. reported that of 737 GIST patients evaluated between 2003 and 2007 in Japan, 24 $(3.3 \%)$ were rectal GISTs (21). All were in the lower rectum, within a median of $2.5 \mathrm{~cm}$ from the anal verge (21). Hamada et al. reported 33 rectal GISTs in Japan before the era of IM. The mean age was 61.6 years, the maximum tumor size was $8.2 \mathrm{~cm}$, and the mean distance from the anal verge was $4.2 \mathrm{~cm} \mathrm{(22).}$

\section{Diagnosis}

Baik et al. reported seven cases of rectal GIST in South Korea with primary symptoms of hematochezia, constipation, and anal pain similar to those of other rectal tumors (10). In a case series in India, the main primary symptoms were pain (38.5\%), bleeding $(23.0 \%)$, and others (38.5\%) (23). Shen et al. described bleeding (28.9\%), pain $(17.8 \%)$, difficulty with defecation $(11.1 \%)$, urinary complaints $(6.7 \%)$, and other symptoms $(11.1 \%)$ in 45 cases of rectal GIST (24). In a patient series in Japan the chief complaints were anal bleeding $(30.3 \%)$, constipation (15.2\%), anal discomfort (12.1\%), palpitation of tumor (12.1\%), abdominal pain (3.0\%), and ischuria (3.0\%) (22). Table 1 summarizes the most common symptoms of rectal GIST, which are primarily bleeding and/or pain; urinary symptoms may occur more frequently than in GISTs at other sites (22-26).

GISTs of the stomach, colon or rectum generally appear as a submucosal mass in endoscopy (27), and are diagnosed in biopsy tissue. In rectal GISTs, immunohistochemical analysis can be CD117 (KIT) positive dominantly, CD34, PDGFRA, smooth muscle actin, S-100, and vimentin positive occasionally (28). Rectal GISTs are classified as very-low risk, low risk, intermediate risk, or high risk tumors by National Institutes of Health (NIH) criteria (29), and the frequency of recurrence has been estimated as $21-100 \%$ for high risk, $0-34 \%$ for intermediate risk, 
Table 2 The recurrent risk in rectal GIST by NIH criteria

\begin{tabular}{|c|c|c|c|c|c|c|}
\hline Authors & Year & No. of patients & Very-low risk & Low risk & Intermediate risk & High risk \\
\hline Liu (31) & 2014 & 21 & $23.8 \%$ & $23.8 \%$ & $28.6 \%$ & $23.8 \%$ \\
\hline Zhou (28) & 2014 & 67 & $0 \%$ & $45.0 \%$ & $34.0 \%$ & $21.0 \%$ \\
\hline Shen (24) & 2015 & 45 & $8.9 \%$ & $22.2 \%$ & $2.2 \%$ & $66.7 \%$ \\
\hline Yasui (21) & 2017 & 24 & $20.8 \%$ & $33.8 \%$ & $0 \%$ & $45.8 \%$ \\
\hline
\end{tabular}

GIST, gastrointestinal stromal tumor; NIH, National Institutes of Health.

$0-45 \%$ for low risk, and $0-23.8 \%$ for very-low risk tumors $(21,24,26,28,30,31)$ (Table 2). A diagnosis of GIST in the rectum was also considered to correlate with poor overall prognosis. However, Fletcher et al. reported that tumor site was not a reliable predictor of outcome (29). One reason for the poor prognosis of rectal GISTs is that the tumor rupture rate is more than four-fold higher than that of nonrectal GISTs (30).

GISTs are usually seen as an exophytic mass that heterogeneously enhances with intravenous contrast because of its high vascularization (32), and contrast enhanced computed tomography (CT) is the standard method of GIST imaging (33). Magnetic resonance imaging (MRI) is useful for liver-specific lesions or patients contraindicated for CT (33). Fluorodeoxyglucose positron emission tomography (FDG-PET) also has good specificity and sensitivity for evaluation of tumor response after IM treatment (32). However, FDG-PET cannot be used to evaluate treatment response if pretreatment FDG-PET was negative. Approximately 20\% of lesions shown on CT do not display appreciable glucose uptake on pretreatment FDG-PET images (32). The imaging characteristics of rectal GISTs have been described by Jiang et al. (34). Enhanced MRI with direct multiplanar capability is useful in determining the exact origin tumor of pelvic tumors, which is often difficult to confirm. The imaging technology using MRI can detect invasion of adjacent organs in greater detail than possible with CT (34).

\section{Treatment}

\section{Surgery}

Surgical resection with curative intent is the standard treatment for localized GIST (35). Complete excision of the tumor is the most significant factor related to outcome, and can be accomplished in $40-60 \%$ of all GIST patients (36). The benefit of histologically negative margins in the surgical treatment of non-metastatic rectal GIST has been confirmed (19). Since GIST may occur anywhere in gastrointestinal tract, the surgical approach varies, with local excision by trans-anal, trans-sacral, or trans-vaginal procedures as the preferred treatments for early, lower rectal GISTs (37). On the other hand, the treatment of advanced rectal GISTs is controversial. Complete curative resection of rectal GISTs is difficult because of its anatomical features (35), and choice of the surgical procedure may be difficult in patients with large tumors close to the anal verge. Rectal GISTs have a high rate of local recurrence regardless of the surgical procedure (21). Surgical treatment is yet to be standardized (20), but local resection, low anterior resection, abdominoperineal resection (APR), and pelvic exenteration are performed. In each procedure, the objective is complete gross resection with negative microscopic margins and without bleeding or rupture of the pseudo capsule (38).

Trans-anal resection is one of the most minimally invasive approaches, but is limited by the distance from the dentate line (39). Trans-coccygeal excision is effective for the lower rectal GISTs, but has high postoperative morbidity, with fistulae occurring in $21 \%$ of patients (40). Matsushima et al. described a trans-coccygeal/trans-sacral approach that is relatively less invasive and recommended it as the treatment of choice for rectal GISTs because proper bowel preparation, prophylactic antibiotics, and adequate drainage reduce postoperative complications such as fistulae (41). Kinoshita $e t$ al. recommended a perineal approach as an option to preserve the anal function in patients with GISTs involving the anterior wall of the lower rectum (42). For small rectal GISTs, local resection may be safe (20). In a series of seven rectal GIST patients with curative 
resection before the IM era (10), two (28.6\%) experienced local recurrence. One patient had undergone Hartmann's procedure for a $12-\mathrm{cm}$ tumor with local recurrence in the rectum. The second, with APR for a $6 \mathrm{~cm}$ tumor experienced local recurrence in the presacral area. Positive resection margins are associated with poorer survival (31), and margins free of tumor cells are most important regardless of the surgical procedure. The need for wide margins is controversial (36). McCarter et al. reported that there was no difference in recurrence-free survival between R0 and R1 margin surgery in GIST (43). Therefore, we should select appropriate surgical procedure from the anus preserving point of view, especially for rectal GIST's patients.

Laparoscopic surgery has been successful for resection of rectal GISTs $(35,44-47)$, including anus-preserving surgery (46). Laparoscopic colorectal surgery is beneficial because of its minimal access trauma (35). Adequate visualization of deep pelvic lesions is possible. Although the data on laparoscopic surgery for rectal GISTs are limited, this approach seems feasible, especially for small tumors (48).

Prognostic factors of rectal GISTs have been identified. In a series of 21 patients, Xiao et al. reported a 5-year overall survival (OS) of $46 \%$, with NIH high risk and hematochezia as independently associated with disease-free survival (DFS) (49). In another series, tumor size $>5 \mathrm{~cm}$ was identified as the most important determinant of survival after surgery; age [hazard ratio (HR), 2.40; 95\% confidence interval (CI), 1.77-3.25], tumor size (HR, 2.24; 95\% CI, 1.35-3.73) were associated with increased mortality (20). Tumor size and mitotic index have also been identified as prognostic factors (50). Gold et al. developed a nomogram to predict 2 - and 5-year recurrence-free survival after curative surgical resection of localized GISTs $(48,50)$. Lymph node dissection is not considered necessary because lymph node metastasis of GISTs is very rare (10).

\section{IM therapy for advanced/metastatic GIST}

IM is a selective inhibitor of transmembrane receptor KIT protein tyrosine kinases. It acts by inhibiting the proliferation of GIST cells that are stimulated by activated KIT receptors $(51,52)$. IM is indicated for the first-line treatment of metastatic or unresectable GIST. An international, large-scale phase II study (B2222) (6) demonstrated that IM was safe and highly effective for advanced GIST. Moreover, a phase II study (STI571B1202) in Japan also found that IM was generally safe for advanced GIST (53). Kanda et al. reported a 5 -year OS of $60.9 \%$ and median survival of 70 months with a median followup of 68 months after IM therapy for advanced GIST, and IM treatment was also well tolerated in Japan (54). Several studies have assessed patients with rectal GIST and have reported that IM therapy showed antitumor effects for advanced rectal GIST and common-site GISTs (19-23).

GISTs share many phenotypic features associated with various KIT and PDGFRA mutations (55). Heinrich et al. described the correlations of kinase genotype and clinical outcome of IM treatment of GIST (CALGB 150105) (56). The objective response rate reported as complete response (CR)/partial response (PR) was $71.7 \%$ with tumors carrying exon 11 mutations, $44.4 \%$ with exon 9 mutations, and $44.6 \%$ with wild-type tumors (56). KIT mutations involving codons 557-558 were reported to have a poor prognosis (57). Andersson et al. found that $57 \%$ of GIST patients had KIT mutations of exon 11, and that approximately $60 \%$ were deletion and $40 \%$ were missense or duplication mutations (55).

The KIT mutation genotypes in rectal GIST are not well known, but have been described in several studies, which are listed in Figure 1 (23-26,58). As seen in GISTs developing at other sites, a large proportion (59-100\%) of rectal GISTs carried exon 11 mutations. We previously characterized the KIT mutations in nine of 12 rectal GIST patients (unpublished data), all of whom were found to have exon 11 mutations. Okamura et al. have confirmed that the exon 11 mutations in colonic GIST are like those present in stomach and small intestinal GIST (59). As exon 11 KIT mutations are the most frequent mutation genotype in rectal GIST, IM can be considered as a first-line treatment of advanced or metastatic rectal GISTs.

Figure 2 shows an enhanced CT image of our patient with rectal GIST. The patient was a 41-year-old woman with a locally advanced rectal GIST. Laparotomy revealed that the tumor was unresectable owing to tumor rupture. The patient underwent IM therapy as primary chemotherapy. The tumor markedly shrank with 18 months of treatment; the maximum diameter changed from 14 to $5 \mathrm{~cm}$, which is a $64.3 \%$ reduction. The patient then underwent secondary surgery and finally achieved complete tumor resection. Histopathological examination of the resected tumor revealed viable tumor cells that accounted for only $20 \%$ of the residual tumor. 


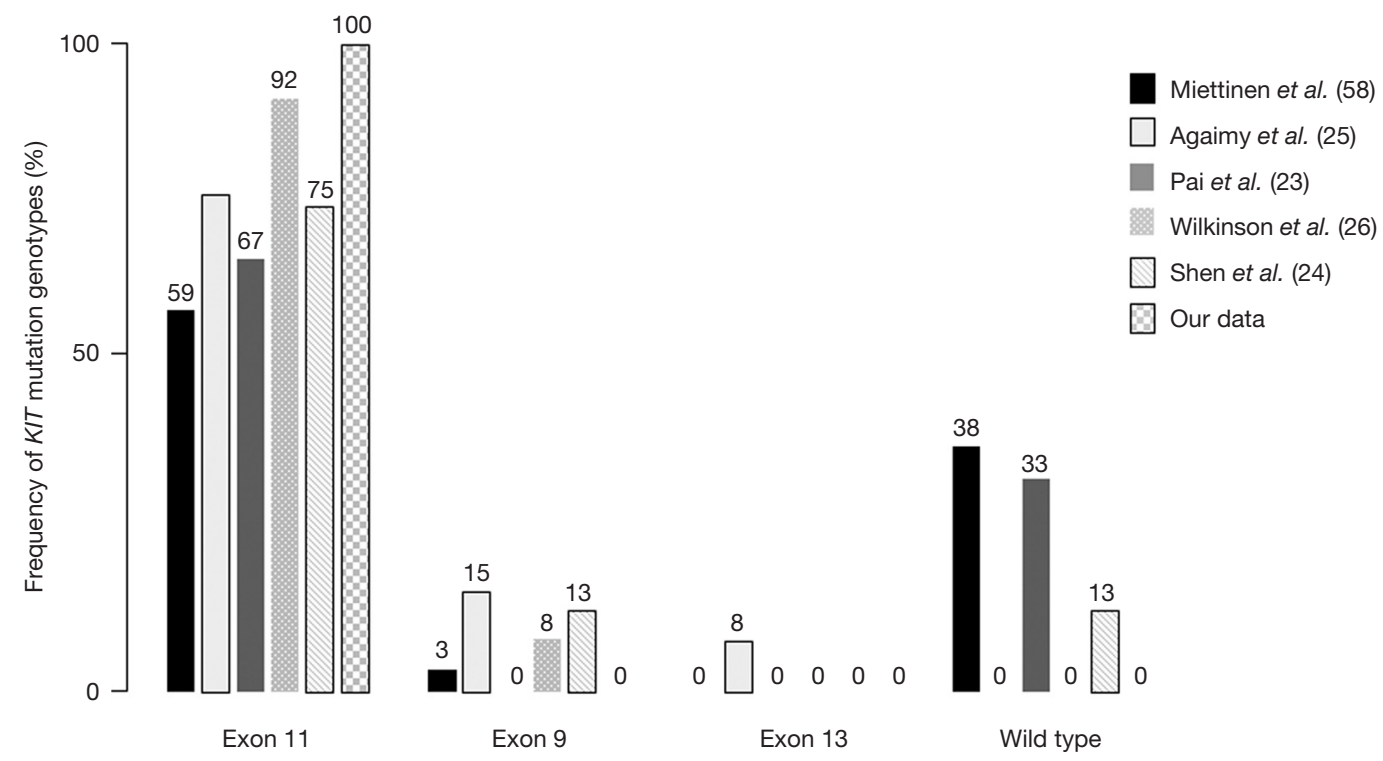

Figure 1 KIT mutation genotypes in rectal GISTs. A large proportion of patients have rectal GISTs with exon 11 mutations. GIST, gastrointestinal stromal tumor.
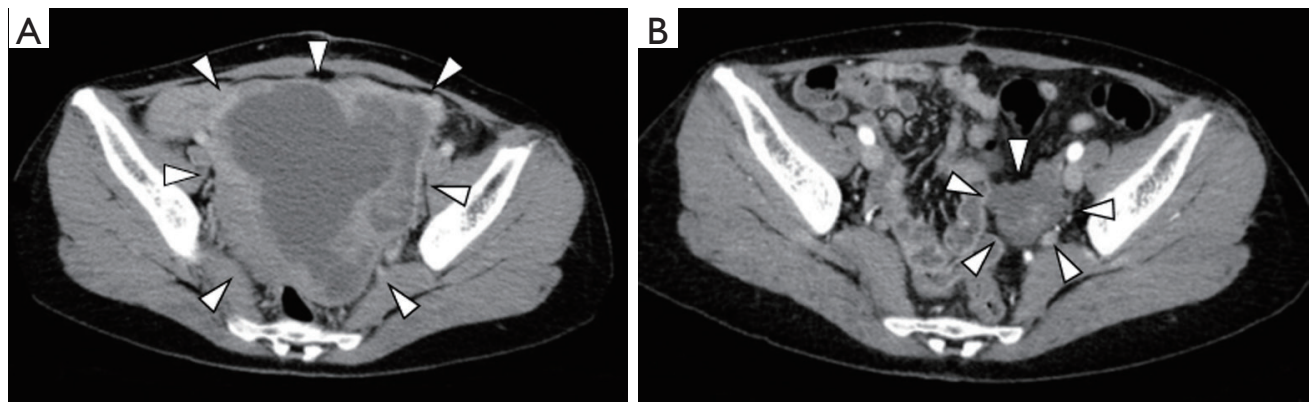

Figure 2 Enhanced pelvic CT scan of a large rectal GIST (A) with maximum diameter of $14 \mathrm{~cm}$ imatinib therapy and (B) $5.0 \mathrm{~cm} 18$ months after initiation of the treatment (arrowheads). GIST, gastrointestinal stromal tumor; CT, computed tomography.

\section{Combined modality therapy for rectal GIST}

Curative resection is an appropriate treatment for rectal GIST, but the recurrent rate is $25 \%$ even in patients with low risk tumor (21). Perioperative treatment with IM may improve outcomes (19-23) and anus-preserving treatment is an important concern because a postoperative stoma decreases a patient's QOL. Tielen et al. reported that preoperative IM led to a decrease in the size of rectal GISTs, but did not lead to less extensive surgery (60). None in a series of seven rectal GIST patients with curative resection before the era of IM experienced anus-preserving surgery (10), and in another series, the anus-preserving rate was $33 \%$ in despite preoperative IM treatment (23). In
Table 3, the anus-preserving rate with rectal GIST is summarized. Before IM treatment, the anus-preserving rate was $14.2 \%$; after IM treatment, the rate was 33.0 $94.9 \%$. Perioperative IM treatment may be promising, but its benefit in anus-preserving surgery in rectal GIST is controversial, and requires further study.

Fujimoto et al. demonstrated the safety and successful use of laparoscopic intersphincteric resection of rectal GIST following IM treatment in a series of five patients (45). The benefits of laparoscopic surgery include an excellent, magnified view in the deep, confined space of the pelvic cavity that enables sphincter and continence conserving surgery $(35,44)$. As there are few reports of the effectiveness 
Table 3 Anus-preserving rate of the surgery for rectal GIST

\begin{tabular}{|c|c|c|c|c|c|c|c|c|}
\hline Authors & Year & $\begin{array}{c}\text { No. of } \\
\text { patients }\end{array}$ & $\mathrm{M} / \mathrm{F}$ & Age & Size (cm) & $\begin{array}{l}\text { Distance from } \\
\text { the anus }(\mathrm{cm})\end{array}$ & $\begin{array}{l}\text { Neoadjuvant } \\
\text { IM }\end{array}$ & $\begin{array}{c}\text { Anus-preserving } \\
\text { rate }\end{array}$ \\
\hline Baik (10) & 2007 & 7 & $2 / 5$ & 54 & 6.6 & 4.1 & $0 \%$ & $14.2 \%$ \\
\hline Jakob (19) & 2013 & 39 & $29 / 10$ & 53 & 5.0 & N/A & $41.0 \%$ & $94.9 \%$ \\
\hline Tielen (60) & 2013 & 32 & $22 / 10$ & 60 & $9.3^{\dagger}, 6.0^{\ddagger}$ & $5.9^{\dagger}, 5.3^{\ddagger}$ & $68.8 \%$ & $37.5 \%$ \\
\hline Pai (23) & 2015 & 13 & $11 / 2$ & 53 & $\mathrm{~N} / \mathrm{A}$ & 2.0 & $100 \%$ & $33.0 \%$ \\
\hline Wilkinson (26) & 2015 & 19 & $11 / 8$ & 57 & 7.6 & N/A & $78.9 \%$ & $84.2 \%$ \\
\hline Yasui (21) & 2017 & 24 & $14 / 10$ & 67 & $\mathrm{~N} / \mathrm{A}$ & 2.5 & $16.7 \%$ & $50.0 \%$ \\
\hline
\end{tabular}

${ }^{\dagger}$, data in patients with imatinib treatment; ${ }^{\ddagger}$, data in patients without imatinib treatment; ${ }^{\S}$, mean. GIST, gastrointestinal stromal tumor; IM, imatinib mesylate; N/A, not applicable.

of laparoscopic resection of rectal GIST following IM treatment, further studies are necessary.

Surgical resection combined with adjuvant IM is expected to improve not only surgical outcome but also survival one. The evidence from randomized trials supports 36 months of adjuvant IM in high risk GIST (61-63). It is important to evaluate the risk of recurrence because rectal GISTs, especially large tumors, have a high risk of recurrence, because of difficulty of curative resection. Tang et al. reported that IM treatment facilitated surgery for very large GISTs, avoided tumor rupture, and was associated with low surgical morbidity (64). In patients with tumors $>5 \mathrm{~cm}, 5$-year mortality in chemotherapy patients (79.2\%) was better than that in patients without chemotherapy (51.2\%, $\mathrm{P}=0.03)$. Hawkins et al. reported that preoperative IM treatment resulted in improved survival of patients with tumors $>5 \mathrm{~cm}$, treated with radical resection (20), but further study is necessary.

The resection with negative margins of rectal GISTs is most important; wide margins are not generally necessary if a non-residual tumor resection is obtained (36). Preoperative IM has been shown to significantly increase the achievement of negative margins and curative resection and to improve local DFS (19). In a series of 36 rectal GIST patients treated with surgery (19), five (13.9\%) developed a local recurrence within a median of 12 months. The patients with local recurrence had not undergone curative resection, and had not received perioperative IM therapy. In another series of 45 patients, Shen et al. reported that DFS of patients with NIH high risk tumors was significantly improved by IM treatment (24), and found that risk category was the only prognostic factor independently associated with DFS (HR, 1.62; 95\% CI, 1.034-2.551). Preoperative IM treatment has also been associated with an increased rate of curative resection, and may facilitate surgical procedures at critical anatomic sites, which have been associated with improved DFS (60) and improved prognosis in rectal GIST (19). The DFS benefit of perioperative IM treatment in patients with intermediate risk and high risk rectal GIST $(\mathrm{P}=0.030)$ was demonstrated by Liu et al. (31). On the other hand, when the preoperative treatment is done, there is a possibility that the preoperative treatment may affect the pathological evaluation of the tumor. In that case, we cannot make the risk evaluation appropriately.

In the era of IM, KIT mutation genotype analysis in pretreatment biopsy samples greatly assists the choice of treatment (19). Genotyping to identify likely nonresponders is important to ensure that a window of opportunity is not missed by delaying surgery in patients who would not benefit from IM or who would benefit from dose escalation (26).

In general, radiotherapy is restricted to symptomatic palliation patient with GIST (65). However, the efficacy of radiotherapy in combination with tyrosine kinase inhibitors for metastatic or advanced GIST has been reported by some researchers $(65,66)$. Ciresa et al. described that the introduction of molecularly targeted therapy combined with radiation therapy could improve the outcomes of patients with GIST (65), but the role of radiotherapy for GIST is controversial. Further studies are warranted to investigate combined modality therapy for rectal GIST patients. 


\section{Conclusions}

Curative resection should be performed for localized rectal GIST. Combined modality therapy, including perioperative IM treatment, is recommended for advanced rectal GIST to facilitate anus-preserving surgery and improve the prognosis. KIT mutation genotype analysis before treatment is important. Further studies of perioperative treatment of patients with rectal GISTs are required to establish an appropriate treatment strategy.

\section{Acknowledgements}

The authors are grateful to Professor Seiichi Hirota, Department of Surgical Pathology, Hyogo College of Medicine, for their assistance with KIT mutation genotype analysis.

\section{Footnote}

Conflicts of Interest: The authors have no conflicts of interest to declare.

\section{References}

1. Mazur MT, Clark HB. Gastric stromal tumors. Reappraisal of histogenesis. Am J Surg Pathol 1983;7:507-19.

2. Downs-Kelly E, Rubin BP. Gastrointestinal stromal tumors: molecular mechanisms and targeted therapies. Patholog Res Int 2011;2011:708596.

3. Joensuu H, Fletcher C, Dimitrijevic S, et al. Management of malignant gastrointestinal stromal tumours. Lancet Oncol 2002;3:655-64.

4. Hirota S, Isozaki K, Moriyama Y, et al. Gain-of-function mutations of c-kit in human gastrointestinal stromal tumors. Science 1998;279:577-80.

5. Hirota S, Ohashi A, Nishida T, et al. Gain-of-function mutations of platelet-derived growth factor receptor alpha gene in gastrointestinal stromal tumors. Gastroenterology 2003;125:660-7.

6. Demetri GD, von Mehren M, Blanke CD, et al. Efficacy and safety of imatinib mesylate in advanced gastrointestinal stromal tumors. N Engl J Med 2002;347:472-80.

7. Lewis JJ, Brennan MF. Soft tissue sarcomas. Curr Probl Surg 1996;33:817-72.

8. Crosby JA, Catton CN, Davis A, et al. Malignant gastrointestinal stromal tumors of the small intestine: a review of 50 cases from a prospective database. Ann Surg
Oncol 2001;8:50-9.

9. Varras M, Vlachakos N, Akrivis C, et al. Malignant gastrointestinal stromal tumor presenting with hemoperitoneum in puerperium: report of a case with review of the literature. World J Surg Oncol 2010;8:95.

10. Baik SH, Kim NK, Lee CH, et al. Gastrointestinal stromal tumor of the rectum: an analysis of seven cases. Surg Today 2007;37:455-9.

11. Tran T, Davila JA, El-Serag HB. The epidemiology of malignant gastrointestinal stromal tumors: an analysis of 1,458 cases from 1992 to 2000. Am J Gastroenterol 2005;100:162-8.

12. Miettinen M, Lasota J. Gastrointestinal stromal tumors (GISTs): definition, occurrence, pathology, differential diagnosis and molecular genetics. Pol J Pathol 2003;54:3-24.

13. Miettinen M, Monihan JM, Sarlomo-Rikala M, et al. Gastrointestinal stromal tumors/smooth muscle tumors (GISTs) primary in the omentum and mesentery: clinicopathologic and immunohistochemical study of 26 cases. Am J Surg Pathol 1999;23:1109-18.

14. Sandrasegaran K, Rajesh A, Rydberg J, et al. Gastrointestinal stromal tumors: clinical, radiologic, and pathologic features. AJR Am J Roentgenol 2005;184:803-11.

15. Nilsson B, Bümming P, Meis-Kindblom JM, et al. Gastrointestinal stromal tumors: the incidence, prevalence, clinical course, and prognostication in the preimatinib mesylate era. A population-based study in western Sweden. Cancer 2005;103:821-9.

16. van der Zwan SM, DeMatteo RP. Gastrointestinal stromal tumor: 5 years later. Cancer 2005;104:1781-8.

17. Tryggvason G, Gíslason HG, Magnússon MK, et al. Gastrointestinal stromal tumors in Iceland, 1990-2003: the icelandic GIST study, a population-based incidence and pathologic risk stratification study. Int J Cancer 2005;117:289-93.

18. Miettinen M, Lasota J. Gastrointestinal stromal tumors: pathology and prognosis at different sites. Semin Diagn Pathol 2006;23:70-83.

19. Jakob J, Mussi C, Ronellenfitsch U, et al. Gastrointestinal stromal tumor of the rectum: results of surgical and multimodality therapy in the era of imatinib. Ann Surg Oncol 2013;20:586-92.

20. Hawkins AT, Wells KO, Krishnamurty DM, et al. Preoperative chemotherapy and survival for large anorectal gastrointestinal stromal tumors: A national analysis of 333 cases. Ann Surg Oncol 2017;24:1195-201. 
21. Yasui M, Tsujinaka T, Mori M, et al. Characteristics and prognosis of rectal gastrointestinal stromal tumors: an analysis of registry data. Surg Today 2017. [Epub ahead of print].

22. Hamada M, Ozaki K, Horimi T, et al. Recurrent rectal GIST resected successfully after preoperative chemotherapy with imatinib mesylate. Int J Clin Oncol 2008;13:355-60.

23. Pai VD, Demenezes JL, Patil PS, et al. Multimodality therapy of rectal gastrointestinal stromal tumors in the era of imatinib-an Indian series. J Gastrointest Oncol 2016;7:262-8.

24. Shen C, Chen H, Yin R, et al. Clinicopathologic, surgical characteristics and survival outcomes of rectal gastrointestinal stromal tumors. Neoplasma 2015;62:610-7.

25. Agaimy A, Vassos N, Märkl B, et al. Anorectal gastrointestinal stromal tumors: a retrospective multicenter analysis of 15 cases emphasizing their high local recurrence rate and the need for standardized therapeutic approach. Int J Colorectal Dis 2013;28:1057-64.

26. Wilkinson MJ, Fitzgerald JE, Strauss DC, et al. Surgical treatment of gastrointestinal stromal tumour of the rectum in the era of imatinib. Br J Surg 2015;102:965-71.

27. Kramp KH, Omer MG, Schoffski P, et al. Sphincter sparing resection of a large obstructive distal rectal gastrointestinal stromal tumour after neoadjuvant therapy with imatinib (Glivec). BMJ Case Rep 2015;2015:bcr2014207775.

28. Zhou Z, Chen Z, Chen M, et al. Clinicopathologic factors predicting outcomes in patients with gastrointestinal stromal tumors of the rectum and colon. Tumour Biol 2014;35:4357-62.

29. Fletcher CD, Berman JJ, Corless C, et al. Diagnosis of gastrointestinal stromal tumors: A consensus approach. Hum Pathol 2002;33:459-65.

30. Farid M, Lee MJ, Chew MH, et al. Localized gastrointestinal stromal tumor of the rectum: An uncommon primary site with prominent disease and treatment-related morbidities. Mol Clin Oncol 2013;1:190-4.

31. Liu H, Yan Z, Liao G, et al. Treatment strategy of rectal gastrointestinal stromal tumor (GIST). J Surg Oncol 2014;109:708-13.

32. Choi H, Charnsangavej C, de Castro Faria S, et al. CT evaluation of the response of gastrointestinal stromal tumors after imatinib mesylate treatment: a quantitative analysis correlated with FDG PET findings. AJR Am J
Roentgenol 2004;183:1619-28.

33. Kalkmann J, Zeile M, Antoch G, et al. Consensus report on the radiological management of patients with gastrointestinal stromal tumours (GIST): recommendations of the German GIST Imaging Working Group. Cancer Imaging 2012;12:126-35.

34. Jiang ZX, Zhang SJ, Peng WJ, et al. Rectal gastrointestinal stromal tumors: imaging features with clinical and pathological correlation. World J Gastroenterol 2013;19:3108-16.

35. Somu K, Dashore AR, Shah AR, et al. Laparoscopic excision of large lower rectal gastrointestinal stromal tumour (GIST): A case report. J Minim Access Surg 2016;12:283-5.

36. Eisenberg BL, Judson I. Surgery and imatinib in the management of GIST: emerging approaches to adjuvant and neoadjuvant therapy. Ann Surg Oncol 2004;11:465-75.

37. Centonze D, Pulvirenti E, Pulvirenti D'Urso A, et al. Local excision with adjuvant imatinib therapy for anorectal gastrointestinal stromal tumors. Tech Coloproctol 2013;17:571-4.

38. Chaudhry UI, DeMatteo RP. Advances in the surgical management of gastrointestinal stromal tumor. Adv Surg 2011;45:197-209.

39. Tazawa H, Hirata Y, Kuga Y, et al. Sphinctersaving resection by cluneal arched skin incision for a gastrointestinal stromal tumor (GIST) of the lower rectum: a case report. Surg Case Rep 2017;3:8.

40. Christiansen J. Excision of mid-rectal lesions by the Kraske sacral approach. Br J Surg 1980;67:651-2.

41. Matsushima K, Kayo M. Transsacral approach to resect a gastrointestinal stromal tumor in the rectum: report of two cases. Surg Today 2007;37:698-701.

42. Kinoshita H, Sakata Y, Umano Y, et al. Perineal approach for a gastrointestinal stromal tumor on the anterior wall of the lower rectum. World J Surg Oncol 2014;12:62.

43. McCarter MD, Antonescu CR, Ballman KV, et al. Microscopically positive margins for primary gastrointestinal stromal tumors: analysis of risk factors and tumor recurrence. J Am Coll Surg 2012;215:53-9.

44. Kyo K, Azuma M, Okamoto K, et al. Neoadjuvant imatinib treatment and laparoscopic anus-preserving surgery for a large gastrointestinal stromal tumor of the rectum. World J Surg Oncol 2016;14:68.

45. Fujimoto Y, Akiyoshi T, Konishi T, et al. Laparoscopic sphincter-preserving surgery (intersphincteric resection) after neoadjuvant imatinib treatment for gastrointestinal stromal tumor (GIST) of the rectum. Int J Colorectal Dis 
2014;29:111-6.

46. Akiyoshi T, Ueno M, Fukunaga Y, et al. Laparoscopic local excision and rectoanal anastomosis for rectal gastrointestinal stromal tumor: modified laparoscopic intersphincteric resection technique. Dis Colon Rectum 2014;57:900-4.

47. Nakamura T, Ihara A, Mitomi H, et al. Gastrointestinal stromal tumor of the rectum resected by laparoscopic surgery: report of a case. Surg Today 2007;37:1004-8.

48. Theodoropoulos DG. Gastrointestinal tumors of the colon and rectum. Clin Colon Rectal Surg 2011;24:161-70.

49. Xiao CC, Zhang S, Wang MH, et al. Clinicopathological features and prognostic factors of rectal gastrointestinal stromal tumors. J Gastrointest Surg 2013;17:793-8.

50. Gold JS, Gönen M, Gutiérrez A, et al. Development and validation of a prognostic nomogram for recurrencefree survival after complete surgical resection of localised primary gastrointestinal stromal tumour: a retrospective analysis. Lancet Oncol 2009; 10:1045-52.

51. Miettinen MM, Sarlomo-Rikala M, Kovatich AJ, et al. Calponin and h-caldesmon in soft tissue tumors: consistent h-caldesmon immunoreactivity in gastrointestinal stromal tumors indicates traits of smooth muscle differentiation. Mod Pathol 1999;12:756-62.

52. Heinrich MC, Corless CL, Demetri GD, et al. Kinase mutations and imatinib response in patients with metastatic gastrointestinal stromal tumor. J Clin Oncol 2003;21:4342-9.

53. Nishida T, Shirao K, Sawaki A, et al. Efficacy and safety profile of imatinib mesylate (ST1571) in Japanese patients with advanced gastrointestinal stromal tumors: a phase II study (STI571B1202). Int J Clin Oncol 2008;13:244-51.

54. Kanda T, Ishikawa T, Hirota S, et al. Prospective observational study of imatinib therapy in Japanese patients with advanced gastrointestinal stromal tumors: long-term follow-up and second malignancy. Jpn J Clin Oncol 2012;42:578-85.

55. Andersson J, Bümming P, Meis-Kindblom JM, et al. Gastrointestinal stromal tumors with KIT exon 11 deletions are associated with poor prognosis. Gastroenterology 2006;130:1573-81.

56. Heinrich MC, Owzar K, Corless CL, et al. Correlation of kinase genotype and clinical outcome in the North American Intergroup Phase III Trial of imatinib mesylate for treatment of advanced gastrointestinal stromal tumor: CALGB 150105 Study by Cancer and Leukemia Group B and Southwest Oncology Group. J Clin Oncol
2008;26:5360-7.

57. Hohenberger P, Ronellenfitsch U, Oladeji O, et al. Pattern of recurrence in patients with ruptured primary gastrointestinal stromal tumour. Br J Surg 2010;97:1854-9.

58. Miettinen M, Furlong M, Sarlomo-Rikala M, et al. Gastrointestinal stromal tumors, intramural leiomyomas, and leiomyosarcomas in the rectum and anus: a clinicopathologic, immunohistochemical, and molecular genetic study of 144 cases. Am J Surg Pathol 2001;25:1121-33.

59. Okamura T, Kanda T, Hirota S, et al. Imatinib therapy for a patient with metastasis of colonic gastrointestinal stromal tumor: report of a case. Clin J Gastroenterol 2013;6:116-21.

60. Tielen R, Verhoef C, van Coevorden F, et al. Surgical management of rectal gastrointestinal stromal tumors. J Surg Oncol 2013;107:320-3.

61. Dematteo RP, Ballman KV, Antonescu CR, et al. Adjuvant imatinib mesylate after resection of localised, primary gastrointestinal stromal tumour: a randomised, doubleblind, placebo-controlled trial. Lancet 2009;373:1097-104.

62. Kanda T, Nishida T, Wada N, et al. Adjuvant therapy with imatinib mesylate after resection of primary high-risk gastrointestinal stromal tumors in Japanese patients. Int J Clin Oncol 2013;18:38-45.

63. Joensuu H, Eriksson M, Sundby Hall K, et al. One vs three years of adjuvant imatinib for operable gastrointestinal stromal tumor: a randomized trial. JAMA 2012;307:1265-72.

64. Tang S, Yin Y, Shen C, et al. Preoperative imatinib mesylate (IM) for huge gastrointestinal stromal tumors (GIST). World J Surg Oncol 2017;15:79.

65. Ciresa M, D'Angelillo RM, Ramella S, et al. Molecularly targeted therapy and radiotherapy in the management of localized gastrointestinal stromal tumor (GIST) of the rectum: a case report. Tumori 2009;95:236-9.

66. Gatto L, Nannini M, Saponara M, et al. Radiotherapy in the management of gist: state of the art and new potential scenarios. Clin Sarcoma Res 2017;7:1.

doi: $10.21037 /$ tgh.2018.01.08

Cite this article as: Kameyama $\mathrm{H}$, Kanda T, Tajima $\mathrm{Y}$, Shimada Y, Ichikawa H, Hanyu T, Ishikawa T, Wakai T. Management of rectal gastrointestinal stromal tumor. Transl Gastroenterol Hepatol 2018;3:8. 